\title{
Episodic volcanism on Enceladus: Application of the Ojakangas-Stevenson model
}

\author{
Jennifer Meyer*, Jack Wisdom \\ Massachusetts Institute of Technology, 54-410 MIT, 77 Massachusetts Avenue, Cambridge, MA 02139, USA
}

\section{A R T I C L E I N F O}

\section{Article history:}

Received 22 August 2007

Revised 19 June 2008

Available online 12 July 2008

\section{Keywords:}

Enceladus

Saturn, satellites

Satellites, dynamics

Resonances, orbital

Tides, solid body

\begin{abstract}
A B S T R A C T
The main equations in the paper "Episodic volcanism of tidally heated satellites with application to Io" by Ojakangas and Stevenson [Icarus 66, 341-358] are presented; numerical integration of these equations confirms the results of Ojakangas and Stevenson [Icarus 66, 341-358] for Io. Application to Enceladus is considered. It is shown that Enceladus does not oscillate about the tidal equilibrium in this model by both new nonlinear stability analysis and numerical integration of the model equations.
\end{abstract}

(C) 2008 Elsevier Inc. All rights reserved.

\section{Introduction}

We have shown that equilibrium tidal heating in Enceladus cannot account for the nonsolar heat emanating from Enceladus (Meyer and Wisdom, 2007): equilibrium tidal heating can account for only $1.1\left(18000 / Q_{s}\right) \mathrm{GW}$ of the observed $5.8 \pm 1.9 \mathrm{GW}$ (Spencer et al., 2006). Provided the origin of the observed heating is tidal heating, it is possible that Enceladus is oscillating about equilibrium. A model for oscillation about equilibrium has been presented for Io by Ojakangas and Stevenson (1986). Fischer and Spohn (1990) presented similar oscillation models for Io, emphasizing different rheologies. Ojakangas and Stevenson (1986) mentioned the possible application of their model to Enceladus.

The Ojakangas and Stevenson model would only apply to Enceladus if heat transport is mainly by convection. Squyres et al. (1983) discuss whether convection occurs in Enceladus. They consider a convecting region overlain by a nonconvecting, conductive crustal ice layer. They find that for crustal thicknesses larger than $30 \mathrm{~km}$, heat transport is dominated by convection. Here we assume convection occurs and that the Ojakangas and Stevenson model is applicable to Enceladus.

We first review the Ojakangas and Stevenson (1986) model. We carry out a new linear stability analysis for their full model. We show that, in fact, Enceladus does not oscillate about the tidal equilibrium within the Ojakangas and Stevenson (1986) model.

\footnotetext{
* Corresponding author.

E-mail addresses: meyerj@mit.edu (J. Meyer), wisdom@mit.edu (J. Wisdom).
}

\section{Ojakangas and Stevenson evolution equations}

Consider the thermal evolution of a satellite, with index 0 , in resonance with another satellite, with index 1 , exterior to it. Let $m_{i}$ be the mass of the satellite $i$, and $n_{i}$ be the mean motion. The semimajor axis of the inner satellite is $a$ and its orbital eccentricity is $e$.

The physical parameters of the inner satellite are the heat capacity $C_{p}$, the temperature $T$, the radius $R$, the density $\rho$, the thermal diffusivity $K$, the surface gravitational acceleration $g$, the thermal expansion coefficient $\alpha_{T}$, the kinematic viscosity $\nu(T)$, the critical Rayleigh number $R a_{c}$, and the Love number $k(T)$ and the tidal quality factor $Q(T)$. The values adopted for these physical parameters are listed in Table 1.

The basic equation for the thermal state states that the rate of change of the thermal energy in the satellite is a balance between tidal heating and loss from thermal convection:

$$
\begin{aligned}
m_{0} C_{p} \frac{d T}{d t}= & \frac{21}{2} \frac{G M_{p}^{2} R^{5} n_{0}}{a^{6}}\left(\frac{k(T) e^{2}}{Q(T)}\right) \\
& -4 \pi R^{2} \rho C_{p} K T\left(\frac{g \alpha_{T} T}{v(T) K R a_{c}}\right)^{1 / 3} .
\end{aligned}
$$

The temperature dependence of the factor $Q / k$ is unknown, but approximated by a power law near the solidus and a constant at low temperature. A form that interpolates these characteristics is

$$
\frac{Q(T)}{k(T)}=f\left(T / T_{m}\right)=\left[\left(\frac{Q}{k}\right)_{0}^{-1}+A\left(\frac{T}{T_{m}}\right)^{n}\right]^{-1},
$$


Table 1

The adopted physical parameters for Io and Enceladus are presented

\begin{tabular}{lll}
\hline & Io & Enceladus \\
\hline$M_{p}\left[10^{24} \mathrm{~kg}\right]$ & 1898.8 & 568.5 \\
$m_{0}\left[10^{20} \mathrm{~kg}\right]$ & 893.3 & 1.08 \\
$m_{1}\left[10^{20} \mathrm{~kg}\right]$ & 479.7 & 10.95 \\
$R_{p}[\mathrm{~km}]$ & 71,492 & 60,330 \\
$Q_{p}$ & $10^{5}$ & $1.8 \times 10^{4}$ \\
$a[\mathrm{~km}]$ & 421,769 & 238,400 \\
$R\left[\mathrm{~km}^{2}\right.$ & 1821.3 & 252.3 \\
$k_{2 p}$ & 0.38 & 0.341 \\
$R a_{c}$ & 800 & 800 \\
$\alpha_{T}\left[\mathrm{~K}^{-1}\right]$ & $3.0 \times 10^{-5}$ & $5.1 \times 10^{-5}$ \\
$K\left[\mathrm{~m}^{2} \mathrm{~s}^{-1}\right]$ & $1.0 \times 10^{-6}$ & $1.35 \times 10^{-7}$ \\
$C_{p}\left[\mathrm{~J} \mathrm{~kg}^{-1} \mathrm{~K}^{-1}\right]$ & 800 & 2100 \\
$T_{m}\left[\mathrm{~K}^{-3}\right]$ & 1400 & 273 \\
$\rho\left[\mathrm{kg} \mathrm{m}^{-3}\right]$ & 3500 & 1602 \\
$g\left[\mathrm{~m} \mathrm{~s}^{-2}\right]$ & 1.8 & 0.11 \\
$k_{2}$ & 0.027 & 0.0018 \\
$\nu_{T M}\left[\mathrm{~m}^{2} \mathrm{~s}^{-1}\right]$ & $10^{12}-10^{13}$ & $10^{10}$ \\
$\beta$ & 13 & 30.7 \\
$\gamma$ & 0.32 & 0.49 \\
$n$ & $20-30$ & $?$ \\
$m$ & $8-12$ & 13 \\
$L$ & $20-30$ & $35-37$ \\
\hline & &
\end{tabular}

with

$A=\left(\frac{Q}{k}\right)_{\min }^{-1}-\left(\frac{Q}{k}\right)_{0}^{-1}$,

where $T_{m}$ is the melting temperature, $(Q / k)_{0}$ is the value of $Q / k$ at low temperature, and $(Q / k)_{\min }$ is the minimum value of $Q / k$ that is reached near the melting temperature. The viscosity is taken as a power law

$\nu(T)=v_{T M}\left(T / T_{m}\right)^{-L}$,

where, in silicates, $20<L<30$, and $v_{T M}=10^{12}-10^{13} \mathrm{~m}^{2} \mathrm{~s}^{-1}$. In water ice, $35<L<37$, and $v_{T M}=10^{10} \mathrm{~m}^{2} \mathrm{~s}^{-1}$ (Durham et al., 1997).

The equilibrium value of the temperature is

$T_{0}=\left[\frac{7}{2 \beta} \frac{k_{p}}{Q_{p}} \frac{G m_{0} R R_{p}^{5} n_{0}}{C_{p} a^{6}}\left(\frac{\nu_{T M} T_{m}^{L} R a_{c}}{g \alpha_{T} K^{2}}\right)^{1 / 3}\right]^{1 / m}$,

where $m=(L+4) / 3$, and $\beta=13$ for Io in the Io-EuropaGanymede resonance and $\beta=30.69$ for Enceladus in the Enceladus-Dione resonance (Meyer and Wisdom, 2007). The equilibrium value of the square of the eccentricity is

$e_{0}^{2}=\frac{m_{0}^{2} R_{p}^{5} k_{p}}{\beta M_{p}^{2} R^{5} Q_{p}} f\left(T_{0} / T_{m}\right)$.

The convective cooling timescale is

$\tau_{t h}=\frac{R}{3}\left(\frac{\nu_{T M} T_{m}^{L} R a_{c}}{g \alpha_{T} K^{2}}\right)^{1 / 3} T_{0}^{1-m}$.

The characteristic timescale for the equilibration of eccentricity near equilibrium is

$\tau_{e}=\frac{m_{1} \alpha|C(\alpha)|}{2 M_{p} \gamma c_{0} e_{0}}$

where $\alpha$ is the semimajor axis ratio $a_{0} / a_{1}, C(\alpha)$ is about -1.19 for a 2:1 mean motion resonance,

$c_{0}=\frac{9}{2}\left(\frac{R_{p}}{R}\right)^{5} \frac{m_{0}}{M_{p}} n_{0} \frac{k_{p}}{Q_{p}}$,

and $\gamma$ is about 0.32 for the Io-Europa-Ganymede resonance. For a two-body $j: j-1$ resonance,

$\gamma=j-1-j \frac{m_{1}}{m_{0}}\left(\frac{a_{0}}{a_{1}}\right)^{8}$.
For the Enceladus-Dione 2:1 resonance, $\gamma=0.49$.

Let

$p=\frac{\tau_{t h}}{\tau_{e}}=\frac{6 \beta}{7} \frac{C_{p} a \gamma}{G m_{1} \alpha|C(\alpha)|} e_{0} T_{0}$.

For Io this constant is about 3 .

Define the nondimensional temperature $T_{N}=T / T_{0}$, the scaled eccentricity $e_{N}=e / e_{0}$, and the nondimensional time $t_{N}=t / \tau_{t h}$. With these definitions the nondimensionalized evolution equations are

$\frac{d T_{N}}{d t_{N}}=\left[\frac{f\left(T_{0} / T_{m}\right)}{f\left(T_{N} T_{0} / T_{m}\right)}\right] e_{N}^{2}-T_{N}^{m}$,

$\frac{d e_{N}}{d t_{N}}=e_{N}^{2} \frac{p}{2}\left[1-\frac{f\left(T_{0} / T_{m}\right)}{f\left(T_{N} T_{0} / T_{m}\right)} e_{N}^{2}\right]$.

The equilibrium heat flow is

$$
\begin{aligned}
q_{0} & =\frac{m_{0} C p}{4 \pi R^{2}} \frac{T_{0}}{\tau_{t h}} \\
& =\frac{7}{2 \beta} \frac{k_{p}}{Q_{p}} \frac{G m_{0} R R_{p}^{5} n_{0} \rho}{a^{6}},
\end{aligned}
$$

which is $0.53\left(100,000 / Q_{J}\right) \mathrm{Wm}^{-2}$ for Io, and $1.48\left(18,000 / Q_{S}\right)$ $\mathrm{mW} \mathrm{m}^{-2}$ for Enceladus. The latter corresponds to a total power of 1.1(18,000/Qs) GW emanating from Enceladus.

In integrating the evolution equations, several assumptions have to be made. The temperature $T$ does not rise above $T_{m}$. During an interval in which $T=T_{m}$, the tidal heating rate is greater than the convective cooling rate, and the excess energy is assumed to be released through volcanism. The interval of $T=T_{m}$ is terminated when the rate of tidal heating falls (due to the declining eccentricity) below the rate of convective cooling. The heat flow during an interval in which $T<T_{m}$ is given by the convective cooling term; the heat flow during an interval in which $T=T_{m}$ is given by the tidal heating term.

In Fig. 1, we show a typical evolution of Io's eccentricity and heat flow in the Ojakangas-Stevenson model. We confirm the oscillating behavior found by Ojakangas and Stevenson (1986). In Fig. 2, we show a typical evolution of Enceladus's eccentricity and heat flow in the same model. Both the eccentricity and heat flow rapidly damp to equilibrium. No oscillations were found for Enceladus.

\section{Stability analysis}

Ojakangas and Stevenson carry out a linear stability analysis for their simplified model in which $Q(T) / k(T)$ is a power law. They then introduce a more realistic form for $Q(T) / k(T)$ that approaches a constant for small $T$ [see Eq. (2)]. However, they do not carry out the stability analysis for this case. Here we describe how the results of the linear stability analysis are modified for their more realistic model.

For the simplified model they found that the equilibrium state, $e_{N}=T_{N}=1$, was unstable if $n>m+p$. Further, they found that there were linear oscillations (either growing or decaying) provided

$m^{2}+n^{2}+p^{2}-2 m p-2 m n-2 n p<0$.

When the tidal equilibrium is linearly unstable, the nonlinear system oscillates.

We have carried out a linear stability analysis for the more realistic model in which $Q(T) / k(T)$ is given by Eq. (2). We find that the results of their analysis for the simplified model continue to hold in the more realistic model if $n$ is replaced by

$n^{\prime}=n A\left(T_{0} / T_{m}\right)^{n} f\left(T_{0} / T_{m}\right)$. 


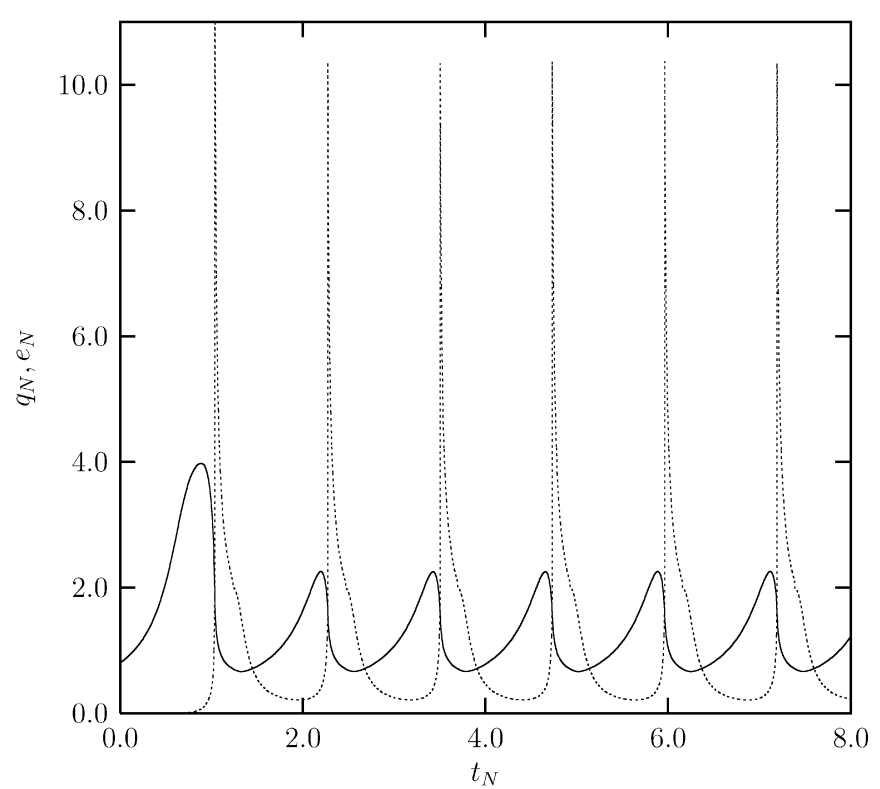

Fig. 1. The scaled eccentricity $e_{N}$ (solid) and the nondimensional heat flow $q_{N}$ (dotted) for Io are plotted versus the nondimensional time. The timescale $\tau_{\text {th }}$ is about $135 \mathrm{Myr}$, the scale for the heatflow is $0.53 \mathrm{~W} \mathrm{~m}^{-2}$, and the scale for the eccentricity is 0.0052 . Here $m=12, n=25,(Q / k)_{0}=200 / 0.027,(Q / k)_{\min }=3 / 0.027$, and $Q_{J}=10^{5}$

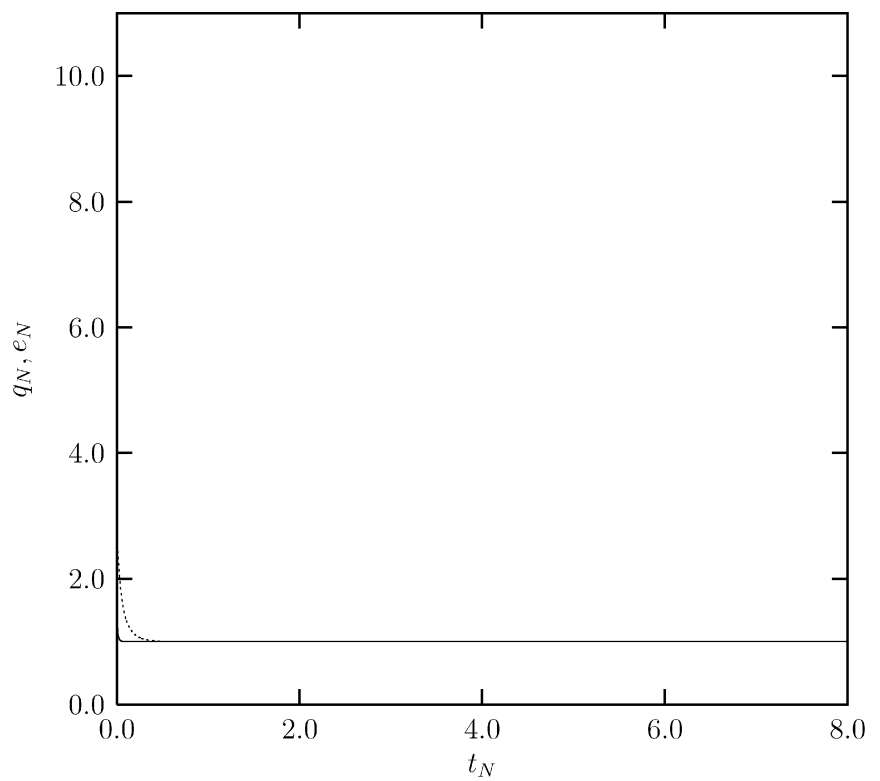

Fig. 2. The scaled eccentricity $e_{N}$ (solid) and the nondimensional heat flow $q_{N}$ (dotted) for Enceladus are plotted versus the nondimensional time.

Thus the equilibrium is linearly unstable if $n^{\prime}>m+p$, and there are linear oscillations (growing or decaying) if

$m^{2}+\left(n^{\prime}\right)^{2}+p^{2}-2 m p-2 m n^{\prime}-2 n^{\prime} p<0$.

For Io, $n^{\prime} \approx n$, for $n$ in the range of interest $20<n<30$, and $T_{0} / T_{m} \approx 0.94$. The system is linearly unstable and develops nonlinear oscillation at moderate $n \approx n^{\prime}$.

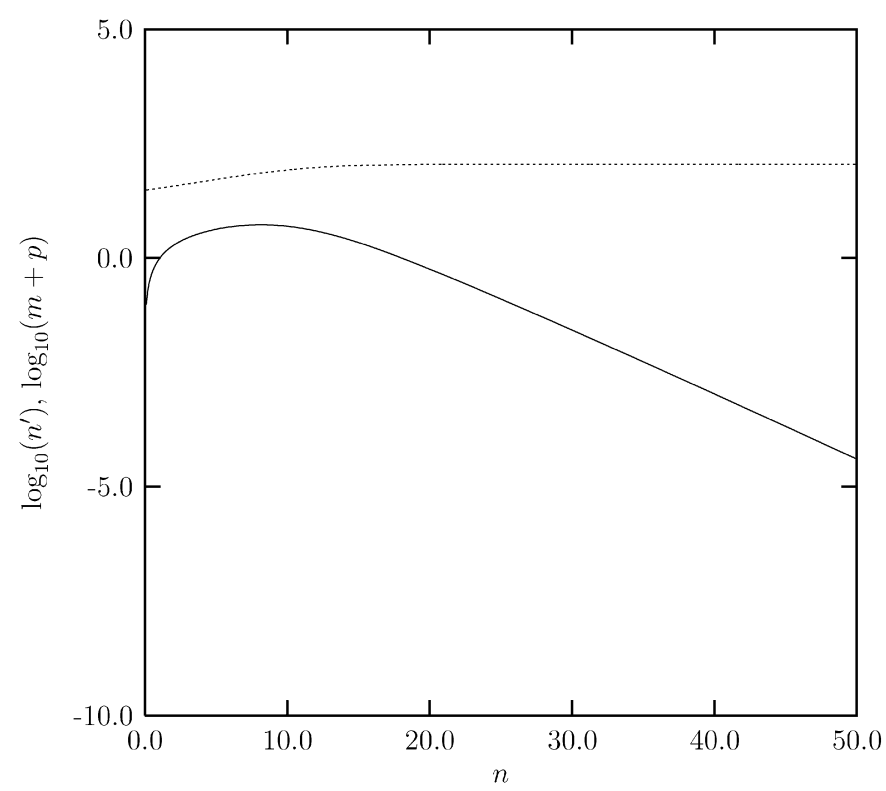

Fig. 3. The parameter $n^{\prime}$ (solid) is plotted as a function of $n$, for Enceladus. This is to be compared with $m+p$ (dotted) plotted as a function of $n$. For $n^{\prime}<m+p$ the system damps to the equilibrium state. The system is stable for all $n$. Here $(Q / k)_{0}$ is $100 / 0.0018 ;(Q / k)_{0}$ is $100 / 0.0018 ; T_{0} / T_{m}=0.70$; and $m=13$.

For Enceladus, $T_{0} / T_{m}$ is smaller $\left(T_{0} / T_{m} \approx 0.70\right)$ so the dropoff in $n^{\prime}$ at large $n$ is more rapid. In fact, the peak of $n^{\prime}$ is about 5 , for $n$ about 8 . At this $n, p \approx 51.2$. Fig. 3 shows a graph of $n^{\prime}$ and $m+p$ as a function of $n$. Enceladus is not in the unstable region for any $n$; instability requires $n^{\prime}>m+p$. This criterion cannot be fulfilled for Enceladus for two reasons: the large value of $p$ requires a large value for $n^{\prime}$ for instability, and the maximum value of $n^{\prime}$ as a function of $n$ is small. Thus for any $n$ the state of Enceladus damps down to the equilibrium state. This conclusion is insensitive to the values we have adopted for the physical parameters.

\section{Conclusion}

We have shown that Enceladus does not oscillate about the tidal equilibrium within the Ojakangas and Stevenson (1986) model. If Enceladus is oscillating about equilibrium, then another model must be developed to describe those oscillations. One possibility is the idea expressed by Yoder (1981) that there might be oscillations about equilibrium if the $Q$ of Enceladus was stress dependent, but this idea has not been developed.

\section{References}

Durham, W.B., Kirby, S.H., Stern, L.A., 1997. Creep of water ices at planetary conditions: A compilation. J. Geophys. Res. 102, 16293-16302.

Fischer, H.-J., Spohn, T., 1990. Thermal-orbital histories of viscoelastic models of Io Icarus 83, 39-65.

Meyer, J.A., Wisdom, J., 2007. Tidal heating in Enceladus. Icarus 188, 535-539.

Ojakangas, G.W., Stevenson, D.J., 1986. Episodic volcanism of tidally heated satellites with application to Io. Icarus 66, 341-358.

Spencer, J.R., Pearl, J.C., Segura, M., Flasar, F.M., Mamoutkine, A., Romani, P., Buratti B.J., Hendrix, A.R., Spilker, L.J., Lopes, R.M.C., 2006. Cassini encounters Enceladus: Background and the discovery of a south polar hot spot. Science 311, 1401-1405. Squyres, S.W., Reynolds, R.T., Cassen, P.M., Peale, S.J., 1983. The evolution of Enceladus. Icarus 53, 319-331.

Yoder, C.F., 1981. Tidal friction and Enceladus' anomalous surface. Eos 62, 939. 